\title{
Effect of Hydrogen on the Stress Relaxation of Aged NiTi Shape Memory Alloys
}

\author{
W. Elkhal LetaieF*, T. Hassine and F. Gamaoun \\ Laboratory of Mechanics of Sousse, The National Engineering School of Sousse, University of Sousse, \\ Hammam Maarouf, Sousse 4054, Tunisia
}

\begin{abstract}
The susceptibility of the NiTi shape memory alloy to relaxation after the hydrogen charging in an aqueous solution has been investigated with respect to ageing during one to six days in air at room temperature. The orthodontic wires have been prepared by immersing in a $0.9 \% \mathrm{NaCl}$ solution for $3 \mathrm{~h}$, under applied current density of $10 \mathrm{~A} / \mathrm{m}^{2}$ and then relaxed with an imposed deformation in a fully austenite state of structure and in a state with $1 / 3$ and $2 / 3$ of the martensite volume fraction. Through the stress relaxation, the hydrogen-charged specimen has shown a significant decrease of the stress, compared to the non-immersed alloy, when the imposed deformation was located in the plateau of the austenite-martensite transformation. It was also found that a longer ageing period is important and the properties of the wires with longer stress relaxation are similar to the those of non-charged wires. Nevertheless, no difference has been detected between the as-received and the as-charged specimens when the imposed deformation was located in the elastic deformation region of the fully austenitic structure. This behavior is attributed to the effect of the gradient of absorbed hydrogen, existing between the surface and the center axis of the studied wires, which facilitates the mobility of the martensite bands during the stress relaxation.
\end{abstract}

DOI: 10.12693/APhysPolA.129.714

PACS/topics: 62.20.FG, 88.30.EM, 87.85.JJ

\section{Introduction}

The NiTi shape memory alloys (SMAs) are considered among the materials which play a main role in medical instruments production due to their biomechanical and biochemical compatibility with the human organism. Its wide usage in the medical field calls for further research, to investigate the impact of different environments on the behavior of the alloy. As an example, there is an important probability for the NiTi orthodontic wire to be affected by the hydrogen in the oral cavity.

Several studies have been carried out to explain the effect of hydrogen on the superelasticity behavior of the $\mathrm{NiTi}$ alloy under various hydrogen concentrations. Indeed, it has been shown that when the amount of absorbed hydrogen exceeds 50-200 mass ppm, an embrittlement will be detected through the austenite-martensite transformation process, This damage does not appear at the elastic deformation of the austenite or the martensite phases [1]. This fracture is attributed to the hindrance by the diffused hydrogen of the twin boundary motion during its nucleation and growth. Hydrogen acts as a barrier for the martensite transformation. Nevertheless, at a low absorbed amount of hydrogen, it has been shown that the superelasticity of the NiTi alloy has not been modified due the extreme mobility of the diffused hydrogen [2].

In our previous work [3], it was indicated that after $8 \mathrm{~h}$ of immersion, the superelasticity of the as-charged $\mathrm{NiTi}$

*corresponding author; e-mail: wissem.khal@gmail.com alloy was not affected. However, after ageing at room temperature in air, the alloy failed in a brittle manner in the plateau of the austenite-martensite transformation. In addition, Tomita et al. [4] have reported that after $2 \mathrm{~h}$ of immersion in $0.9 \% \mathrm{NaCl}$ solution, and after subsequent $240 \mathrm{~h}$ ageing in air, hydrogen did not diffuse out from the specimen. Consequently, we have attributed this damage to the hydrogen diffusion into the entire volume of the wire, in the weak trapped activation energy sites, such as vacancies and strain field of dislocations, which facilitate a brittle fracture.

In this work, a preliminary research investigating the effect of ageing on the relaxation mechanism of a cathodically-charged NiTi orthodontic wires is presented. The effect of the martensite volume fraction during the relaxation process is also discussed.

\section{Experimental procedure}

Specimens with the nominal composition of 50.8 at.\% $\mathrm{Ni}$ and 49.2 at.\% $\mathrm{Ti}$ have been used in the present study. The samples have a wire shape with a gage length of $20 \mathrm{~mm}$ and a cross section of $0.43 \times 0.64 \mathrm{~mm}^{2}$. The transformation temperatures, namely Ms (the martensite transformation start temperature), Mf (the martensite transformation finish temperature), As (the austenite transformation start temperature) and Af (the austenite transformation finish temperature), have been measured by differential scanning calorimetry (DSC). Their respective values are $-9{ }^{\circ} \mathrm{C},-32{ }^{\circ} \mathrm{C},-11^{\circ} \mathrm{C}$ and $-3{ }^{\circ} \mathrm{C}$. All tensile tests have been done at room temperature in air with a low strain rate of $10^{-4} \mathrm{~s}^{-1}$ using an uniaxial tensile loading Instron 5566-type machine with a load cell of $10 \mathrm{kN}$. To study 
the effect of the martensite phase, the relaxation tests have been conducted for $12 \mathrm{~h}$ at different imposed deformations. The initial applied loading corresponds to an imposed strain of $1.5 \%$ (during the elastic deformation of the austenite phase), $4.0 \%$ (corresponds to $1 / 3$ of the martensite transformation) and $6.3 \%$ (corresponds to $2 / 3$ of the martensite transformation). In order to study the effect of hydrogen on the relaxation mechanism of the NiTi alloy, the wires have been charged electrolytically with hydrogen in the $0.9 \% \mathrm{NaCl}(0.15 \mathrm{NaCl})$ aqueous solution for $3 \mathrm{~h}$ with a current density of $10 \mathrm{~A} / \mathrm{m}^{2}$. Such period of hydrogen charging corresponds to the critical amount of diffused hydrogen, at which the critical stress of austenite-martensite transformation has been kept the same as the one of the non-charged sample. To study the effect of the distribution of hydrogen on the relaxation mechanism of the NiTi alloy, the relaxation tests have been carried out immediately after immersion in sodium chloride solution, and after ageing during $24 \mathrm{~h}$ to $96 \mathrm{~h}$.

\section{Results and discussion}

Figure 1a shows a typical engineering stress-strain curve of the non-charged NiTi wire at room temperature with an imposed strain rate of $10^{-4} \mathrm{~s}^{-1}$. Upon loading, we have found the elastic deformation of the fully austenite phase, followed by a plateau of the austenitemartensite transformation until about $8.5 \%$ of strain, and then an elastic deformation of the produced martensite phase. After $3 \mathrm{~h}$ of immersion in the sodium chloride solution under a current density of $10 \mathrm{~A} / \mathrm{m}^{2}$, the tensile curve shows that the hydrogen does not affect notably the mechanical behavior of the alloys. However, we notice that the hardening-like slope of the phase-transformation pseudo-plateau grows slightly and a minor reduction of the plateau is detected. According to our previous results [5-7], this could be attributed to the hindrance of the austenite transformation by the absorbed hydrogen. In addition, this result indicates that after $3 \mathrm{~h}$ of immersion, the amount of diffused hydrogen is not enough to cause an increase in the critical stress for introducing martensite or to generate an embrittlement during the austenite transformation.

The results of relaxation tests obtained at different imposed deformations are shown in Fig. 1b. It can be seen that in the first stage of the relaxation, the stress promptly drops from the initial stress and gets stabilized at the equilibrium stress value. In addition, it is noticed that the difference between the initial and the equilibrium stress is almost similar when the imposed deformation is localized in the plateau of the austenite-martensite deformation. For an imposed deformation of $4.0 \%$ and $6.3 \%$ this difference is about $25 \mathrm{MPa}$. This result points out that the amount of the produced martensite does not affect the relaxation mechanism of the NiTi wires. Very recently [8], our study on the strain rate response of a superelastic NiTi alloy after hydrogen charging, has indicated that for a strain rate lower than $10^{-3} \mathrm{~s}^{-1}$, a limited

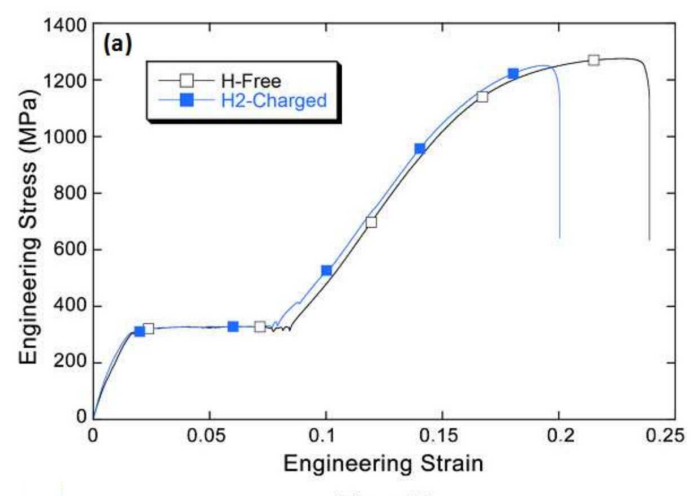

Time (s)

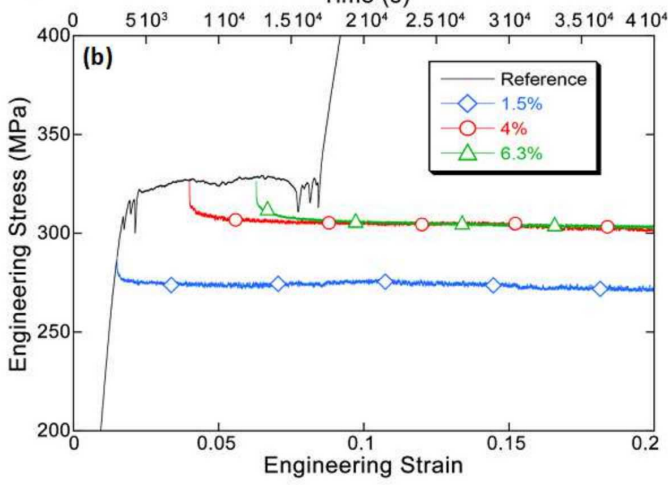

Fig. 1. (a) Typical stress-strain curves of H-free (open squares) and H-charged (solid squares) NiTi wires at a strain rate of $10^{-4} \mathrm{~s}^{-1}$. (b) Superposition of the relaxation curves of the $\mathrm{H}$-free $\mathrm{NiTi}$ at different imposed strains: $1.5 \%$ (open rhombus), $4 \%$ (open circles) and $6.3 \%$ (open triangles).

number of the introduced martensite variants grow under the applied load. In addition, these variants do not increase during the austenite-martensite plateau. Under dynamical ac and dc tensile testing, Fan et al. [9] have shown that the relaxation peak was due to the displacement of the austenite martensite interfaces under an applied stress. Consequently, in our case, it is assumed that with an imposed deformation of $4.0 \%$ and $6.3 \%$, the generated martensite bands are almost similar, and for the both imposed strains, the relaxation mechanism is governed by the same number of austenite-martensite interfaces. This could explain why the difference between the initial and the equilibrium stress during the plateau of the austenite martensite transformation is almost equivalent.

After $3 \mathrm{~h}$ of immersion in $0.9 \% \mathrm{NaCl}$ aqueous solution under a current density of $10 \mathrm{~A} / \mathrm{m}^{2}$ at room temperature, the relaxed stress with an imposed deformation in the elastic deformation of the austenite phase ( $1.5 \%$ of strain) is similar to the non-charged one. Indeed, the difference between the initial and the equilibrium stress is about $17 \mathrm{MPa}$, which is equal to the value obtained for the as-received alloy. As a consequence, it has been shown that after $3 \mathrm{~h}$ of immersion the quantity of the absorbed hydrogen does not affect the relaxation of the fully austenite structure. In contrast, for 


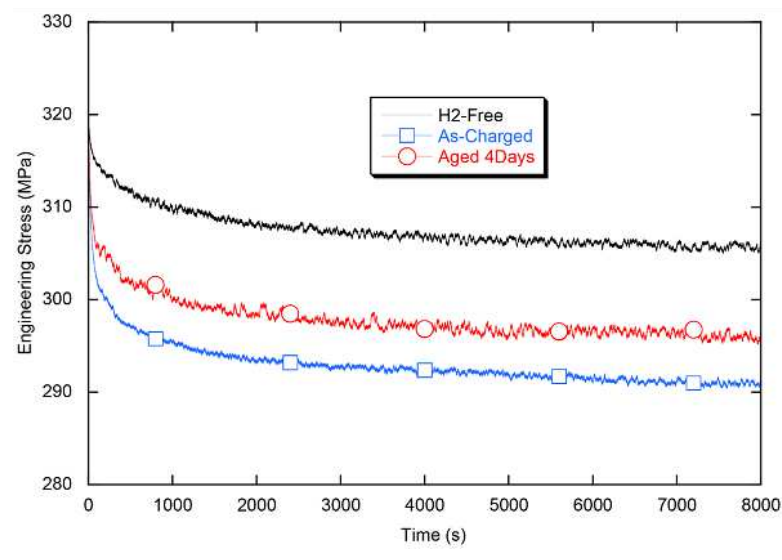

Fig. 2. Relaxation curves, with an imposed deformation of $6.3 \%$, of the non-charged (continuous line), the as-immersed (open squares) and the charged and aged during 4 days (open circles)specimens.

the as-received and charged wires, the relaxation curves, which have been carried out with an imposed deformation located in austenite-martensite plateau, show that the decrease in the stress is higher than in the nonimmersed ones. In fact, the difference between the initial and the equilibrium stress is about $25 \mathrm{MPa}$ for the asreceived wire. Thus for the as-charged alloy the difference is about $40 \mathrm{MPa}$ (Fig. 2). Moreover, for the charged and aged during 4 days specimen, the equilibrium stress is located between the two previous values, and the stress decreases to about $35 \mathrm{MPa}$. These results show that the ageing has a significant effect on the relaxation mechanism of the NiTi alloy after hydrogen charging.

Yokoyama et al. [10] have reported that after cathodical charging, the superelastic NiTi wires present a high hydrogen concentration at the surface. In addition, it has been indicated that the superelastic NiTi alloy brittles during the martensite transformation, and this damage is attributed to the diffusion of the hydrogen from the surface to the entire sample during ageing. Moreover, our study on the ageing effect and the strain rate dependency of the equi-atomic NiTi alloys on the strain rates after hydrogen charging [3] has pointed out that under an applied load with a low strain rate in the specimens immersed for $8 \mathrm{~h}$, the diffused hydrogen of the as-charged alloy has enough time to diffuse from the surface to the center by the so-called "stress-induced diffusion" process, to cause fracture.

In this study, we have shown that, for a low strain rate of $10^{-4} \mathrm{~s}^{-1}$, the stress relaxation of the as-charged specimens is higher than that of the non-charged one. In addition, it has been indicated that the equilibrium stress has a tendency of reaching the stress value of the non-immersed alloy. These results suggest that there is a dependence between the diffusion mechanism of hydrogen, from the surface to the entire specimen, and the relaxation process. Besides, the DMA results [11] have indicated that, at room temperature, the diffused hydrogen can always follow the motion of the twin boundary of the NiTi materials during its relaxation. Consequently, it is assumed that during relaxation, the diffusion of hydrogen facilitates the motion of the austenite-martensite bands. This assumption is due to the fact that, immediately after hydrogen charging, the absorbed hydrogen, which is located in the sub-surface of the specimen, diffuses from the surface to the center to equilibrate its concentration. As a result, during the stress relaxation, the variant interface mobility increases under the permanent loading. In addition, after ageing, the contribution of hydrogen to the austenite-martensite interface displacement goes down, since the gradient of absorbed hydrogen, between the surface and the center, is less pronounced [3]. This suggestion could explain the decrease in the difference between the initial and the equilibrium stress after ageing.

\section{Conclusions}

In this study we have investigated the effect of ageing on the relaxation mechanism of the superelastic $\mathrm{NiTi}$ alloy after $3 \mathrm{~h}$ of hydrogen charging in a $0.9 \% \mathrm{NaCl}$ aqueous solution with a current density of $10 \mathrm{~A} / \mathrm{m}^{2}$ at room temperature. It has been shown that immediately after hydrogen charging the stress relaxation, with an imposed deformation in the austenite-martensite plateau, is higher than that of the non-charged specimen. Furthermore, it has been indicated that after ageing the equilibrium stress has a tendency to reach that of the nonimmersed alloy. This behavior is attributed to activation of the mobility of the martensite bands, under the stress relaxation, by the diffusion of absorbed hydrogen from the surface into the entire bulk of the alloy.

\section{References}

[1] K. Yokoyama, T. Ogawa, K. Takashima, K. Asaoka, J. Sakai, Mater. Sci. Eng. A 466, 106 (2007).

[2] A. Moitra, K.N. Solanki, M.F. Horstemeyer, Comp. Mater. Sci. 50, 820 (2011).

[3] F. Gamaoun, T. Hassine, J. Alloy. Compd. 615, 680 (2014).

[4] M. Tomita, K. Yokoyama, K. Asaoka, J. Sakai, Mater. Sci. Eng. A 476, 308 (2008).

[5] F. Gamaoun, M. Ltaief, T. Bouraoui, T. Ben Zineb, J. Intel. Mat. Syst. Str. 22, 2053 (2011).

[6] F. Gamaoun, I. Skhiri, T. Bouraoui, T. Ben Zineb, J. Intel. Mat. Syst. Str. 25, 980 (2014).

[7] F. Gamaoun, I. Skhiri, T. Bouraoui, Adv. Mat. Res. 324, 181 (2011).

[8] F. Gamaoun, T. Hassine, T. Bouraoui, Phil. Mag. Lett. 94, 30 (2014).

[9] G. Fan, Y. Zhou, K. Otsuka, X. Ren, K. Nakamura, T. Ohba, T. Suzuki, I. Yoshida, F. Yin, Acta Mater. 54, 5221 (2006).

[10] K. Yokoyama, M. Tomita, J. Sakai, Acta Mater. 57, 1875 (2009).

[11] G. Fan, K. Otsuka, X. Ren, F. Yin, Acta Mater. 56, 632 (2008). 